\title{
Bakkenolide-IIIa ameliorates lipopolysaccharide-induced inflammatory injury in human umbilical vein endothelial cells by upregulating LINC00294
}

\author{
JICHONG XU* , HAO FENG ${ }^{*}$, LIN MA, HUAQIAO TAN, SHUO YAN and CHUN FANG \\ Department of Interventional Radiology, Tongji Hospital of Tongji University, Shanghai 200065, P.R. China
}

Received August 27, 2020; Accepted February 4, 2021

DOI: $10.3892 / \mathrm{mmr} .2021 .12016$

\begin{abstract}
Inflammation, which causes injury to vascular endothelial cells, is one of the major factors associated with atherosclerosis (AS); therefore, inhibition of endothelial inflammation is a key step toward preventing AS. The present study aimed to investigate the effects of bakkenolide-IIIa (Bak-IIIa), an important active component of bakkenolides, on endothelial inflammation, as well as the mechanisms underlying such effects. Lipopolysaccharide (LPS)-dama ged human umbilical vein endothelial cells (HUVECs) were treated with Bak-IIIa. The results of the MTT assay and enzyme-linked immunosorbent assay indicated that Bak-IIIa significantly alleviated survival inhibition, and decreased the levels of LPS-induced TNF- $\alpha$, interleukin (IL)- $1 \beta$, IL-8, and IL-6. Furthermore, long noncoding RNA (lncRNA) microarray analyses revealed 70 differentially expressed lncRNAs (DELs) in LPS-damaged HUVECs treated with Bak-IIIa. lncRNA target prediction results revealed that 44 DELs had 52 cis-targets, whereas 12 DELs covered 386 trans-targets. Gene Ontology (GO) and Kyoto Encyclopedia of Genes and Genomes analyses of the trans-targets indicated that three GO terms were associated with inflammation. Therefore, 17 targets involved in these GO terms and six relevant DELs were screened out. Validation via reverse transcription-quantitative PCR indicated that the fold change of NR_015451 (LINC00294) was the highest among the six candidates and that overexpression of LINC00294 significantly alleviated LPS-induced survival inhibition and inflammatory damage in HUVECs. In conclusion, Bak-IIIa ameliorated LPS-induced inflammatory damage in HUVECs by upregulating
\end{abstract}

Correspondence to: Dr Chun Fang or Dr Shuo Yan, Department of Interventional Radiology, Tongji Hospital of Tongji University, 389 Xincun Road, Shanghai 200065, P.R. China

E-mail: fang1972@vip.sina.com

E-mail: 149534145@qq.com

*Contributed equally

Key words: endothelial inflammation, bakkenolide-IIIa, LINC00294
LINC00294. Thus, Bak-IIIa exhibited potential for preventing vascular inflammation.

\section{Introduction}

Atherosclerosis (AS) is the main cause of cardiovascular diseases and is a chronic progressive disease initiated by vascular inflammation $(1,2)$. The endothelium is the innermost layer of blood vessels and changes in blood environment serve an important role in vascular homeostasis. Several factors, such as hyperlipidemia, tobacco use, hypercysteinemia, diabetes and hypertension $(3,4)$, may induce vascular injury by activating endothelial cells, which then release increased amounts of proinflammatory cytokines, chemokines and adhesion molecules $(5,6)$. Subsequently, circulating monocytes are recruited to the lesion site, further triggering a series of pathogenic processes, including excessive lipid accumulation, tunica smooth muscle cell migration and plaque formation (7). Therefore, inhibition of endothelial inflammatory processes may constitute a key step toward preventing AS.

Bakkenolides are a relatively rare class of natural products, which can be isolated from plants of the genus Petasites, including Petasites japonicus (Sieb. et Zucc.) F. Schmidt and Petasites tricholobus Franch (8), which have been used in Chinese folk medicine to treat palsy, hypertension, cough and snakebite for hundreds of years (9). Compared with three other bakkenolides, bakkenolide-IIIa (Bak-IIIa), which is an important active component of bakkenolides, has been reported to display the best neuroprotective effect against oxygen-glucose deprivation-induced neuronal injuries (9). Another study involving animal cerebral damage models indicated that Bak-IIIa could exert neuroprotective effects by inhibiting nuclear factor (NF)- $\kappa \mathrm{B}$ activation (10). Inflammation is a fundamental, yet complex, biological response initiated by the body to counter injuries, as well as infections, and to eliminate the initial causes of cell injury (11). Although the inflammatory levels of injured neurons were not evaluated in the two aforementioned studies, it is reasonable to surmise that inflammation may have been decreased, considering the significant neuroprotective effect of Bak-IIIa.

Long noncoding RNAs (lncRNAs) belong to an important class of ncRNAs that are involved in the regulation of various physiological and pathological processes, including AS (12). Recently, some lncRNAs have been identified as 
novel regulators of vascular inflammation, which constitutes an initial step toward AS $(12,13)$. For example, the lncRNA HOXA-AS3 has been revealed to be a critical activator that promotes NF- $\kappa \mathrm{B}$-mediated endothelial inflammation (14). In addition, the IncRNA H19 was upregulated in the serum of patients with AS and in oxidized low-density lipoprotein (ox-LDL)-treated human umbilical vein endothelial cells (HUVECs), whereas knockdown of its expression significantly ameliorated inflammation, apoptosis and oxidative stress in ox-LDL-injured HUVECs (15). In addition, the lncRNAs HOXA-AS2 and MALAT1 have been shown to participate in the regulation of endothelial inflammation $(16,17)$.

The present study investigated the effect of Bak-IIIa on lipopolysaccharide (LPS)-induced inflammatory injury in HUVECs and identified potential lncRNAs involved in this process. The present study may provide a candidate molecule for the treatment of AS.

\section{Materials and methods}

Cells and cell culture. HUVECs were purchased from American Type Culture Collection, and were cultured in DMEM supplemented with $10 \%$ fetal bovine serum and $1 \%$ penicillin-streptomycin (all from Gibco; Thermo Fisher Scientific, Inc.) at $37^{\circ} \mathrm{C}$ in a humidified atmosphere containing $5 \% \mathrm{CO}_{2}$.

Cell viability assay. HUVECs were seeded in a 96-well plate at 8,000 cells/well. The next day, the cells were exposed to different concentrations $(0,10,20,50,100$ and $200 \mu \mathrm{M})$ of Bak-IIIa (CSNpharm, Inc.) for $72 \mathrm{~h}$ at $37^{\circ} \mathrm{C}$. The supernatant was then removed, and $100 \mu \mathrm{l}$ medium containing $50 \mu \mathrm{g}$ MTT was added to the wells. After incubation at $37^{\circ} \mathrm{C}$ for $3 \mathrm{~h}$, accumulated formazan was dissolved in $100 \mu \mathrm{l}$ DMSO (Sangon Biotech Co., Ltd.), and absorbance was determined at $490 \mathrm{~nm}$ using a microplate reader (ELx800; BioTek Instruments, Inc.).

To observe the effect of Bak-IIIa on LPS-induced cellular injury, HUVECs were first exposed to $10 \mathrm{ng} / \mathrm{ml} \mathrm{LPS}$ (Sigma-Aldrich; Merck KGaA) for $4 \mathrm{~h}$ at $37^{\circ} \mathrm{C}$, and then treated with $0,10,20$ and $50 \mu \mathrm{M}$ Bak-IIIa for $24 \mathrm{~h}$ at $37^{\circ} \mathrm{C}$. Cell survival was assessed using the aforementioned MTT assay. Cells treated without LPS and Bak-IIIa were used as the control.

Enzyme-linked immunosorbent assay (ELISA). HUVECs were seeded in a 12 -well plate at $3 \times 10^{4}$ cells/well, and were treated with LPS for $4 \mathrm{~h}$, and with 0, 10, 20 and $50 \mu \mathrm{M}$ Bak-IIIa for $24 \mathrm{~h}$, respectively. Thereafter, cell culture supernatants were collected and filtered through a $0.45-\mu \mathrm{m}$ cell Nalgene syringe filter (Thermo Fisher Scientific, Inc.). TNF- $\alpha$ (cat. no. PT518), interleukin (IL)-1 $\beta$ (cat. no. PI305), IL-8 (cat. no. PI640) and IL-6 (cat. no. PI330) levels in the supernatants were analyzed using corresponding ELISA kits (Beyotime Institute of Biotechnology), according to the manufacturer's instructions.

Microarray analysis. After LPS-treated HUVECs were treated with 0 or $50 \mu \mathrm{M}$ Bak-IIIa for $24 \mathrm{~h}$ (named as LPS or Bak-IIIa group respectively), total RNA from these two groups was extracted using TRIzol ${ }^{\circledR}$ reagent (Invitrogen; Thermo Fisher Scientific, Inc.). Subsequent microarray hybridization and data analysis were performed by Bhbio Ltd. Co (http://www.shbio.com/). Briefly, purified RNA from each sample was amplified and labeled using a Low Input Quick Amp Labeling kit (Agilent Technologies, Inc.). Thereafter, labeled cRNA was hybridized in a hybridization oven (Agilent Technologies, Inc.), and the hybridized slides were washed and scanned using a Microarray Scanner (Agilent Technologies, Inc.). Raw data were extracted using Feature Extraction software (v10.7.1.1; Agilent Technologies, Inc.) and normalized via a quantile algorithm. Differentially expressed lncRNAs (DELs) were filtered through fold change absolute values (FC abs $\geq 2)$ and the significance of $\mathrm{P}$-values $(\mathrm{P}<0.5)$.

Prediction of IncRNA targets. For predicting cis-regulation, genes transcribed within $10 \mathrm{~kb}$ upstream or downstream of DELs were considered as $c i s$-targets. DELs and potential cis-targets were paired using the UCSC genome browser (18). For trans-target prediction, complementary or similar sequences were first selected via BLAST software (https://ftp.ncbi. nlm.nih.gov/blast/executables/blast+/LATEST/). Thereafter, complementary energy between the two sequences was calculated using RNAplex software (v2.1.8) (19) and energy $\leq-30$ was selected as the trans-target of DELs.

Bioinformatics analyses. Gene Ontology (GO) and Kyoto Encyclopedia of Genes and Genomes (KEGG) analyses (https://david.ncifcrf.gov/) were conducted to determine enrichment of the predicted targets of DELs. Both cis- and trans-targets were first classified into corresponding GO terms or KEGG pathways according to each database, and the top 30 GO terms or KEGG pathways showing a higher enrichment factor and a significant P-value $(<0.5)$ were selected.

Reverse transcription-quantitative PCR (RT-qPCR) analysis. Total RNA was isolated from cells in the LPS and Bak-IIIa groups using TRIzol ${ }^{\circledR}$ reagent (Thermo Fisher Scientific, Inc.) and cDNA was synthesized using PrimeScript ${ }^{\mathrm{TM}}$ One Step RT-PCR kit (Takara Biotechnology Co., Ltd.), according to the manufacturer's protocol.RT-qPCR was performed using SYBR Green PCR Master Mix (Applied Biosystems; Thermo Fisher Scientific, Inc.), following the manufacturer's instructions. Thermocycling was: $94^{\circ} \mathrm{C}$ for $5 \mathrm{~min}$, then 40 cycles at $94^{\circ} \mathrm{C}$ for $5 \mathrm{sec}$ and $60^{\circ} \mathrm{C}$ for $1 \mathrm{~min}$. Expression levels were calculated using the $2^{-\Delta \Delta \mathrm{Cq}}$ method (20), and relative levels of lncRNA were normalized to GAPDH. Primer sequences for lncRNAs and GAPDH are listed in Table I.

Transfection assay. HUVECs were seeded in 6-well plates $\left(2 \times 10^{5}\right.$ cells/well $)$ and transfected with $2 \mu \mathrm{g}$ pCDH-CMV-MCS-EF1-GFP-T2A-Puro plasmid encoding full length LINC00294 (Hanbio Biotechnology Co., Ltd.) or the empty vector, using Lipofectamine ${ }^{\circledR} 2000$ (Invitrogen; Thermo Fisher Scientific, Inc.), following the manufacturer's instructions. After transfection at $37^{\circ} \mathrm{C}$ for $48 \mathrm{~h}$, cells were collected for RT-qPCR validation. HUVECs transfected with the plasmid or empty vector were categorized as LINC00294 or negative control (NC) groups, respectively and were then used for LPS treatment, followed by cell viability and ELISA assay.

Statistical analysis. SPSS v16.0 (SPSS, Inc.) was used for data processing. Data are presented as the mean \pm standard deviation and each experiment was performed three times. Differences 
Table I. Primer sequences for reverse transcription-quantitative PCR.

\begin{tabular}{llc}
\hline LncRNA/Gene & Forward $\left(5^{\prime} \rightarrow 3^{\prime}\right)$ & Reverse $\left(5^{\prime} \rightarrow 3^{\prime}\right)$ \\
\hline NONHSAT089168 & TGTCATTCAGCCCAAACTCA & CGCCAGGTTAGTTCAACACA \\
NR_015451 & TCTCGATCTCCTGACCTCGT & ACAGAGCAGCCTCCACCTTA \\
NR_038275 & CCCTCCTATGTGCTCGTTTC & GAGAAGTCCTCGTCGGTCAG \\
lnc-MUC20-9:1 & GCCTGTCTCTGCCTCTGAAC & CCAGGCCAGACCAATCTAAA \\
NR_034072 & TCCCAAAGTGCTGGGATTAC & CAGTTGGGAACTTGGGAAAA \\
lnc-HSP90B1-3:1 & ACTTGAGGCCAGGAGTTTGA & AACCTCTCAGGCTCAAGCAA \\
GAPDH & CGACCACTTTGTCAAGCTCA & AGGGGAGATTCAGTGTGGTG
\end{tabular}

lncRNA, long non-coding RNA.<smiles>[CH][C@]12[C@@H](OC(=O)/C=C\SC)CC[C@H](C)[C@]1(C)C[C@@]1(C(=C)COC1=O)[C@H]2OC(=O)/C(C)=C\C</smiles>
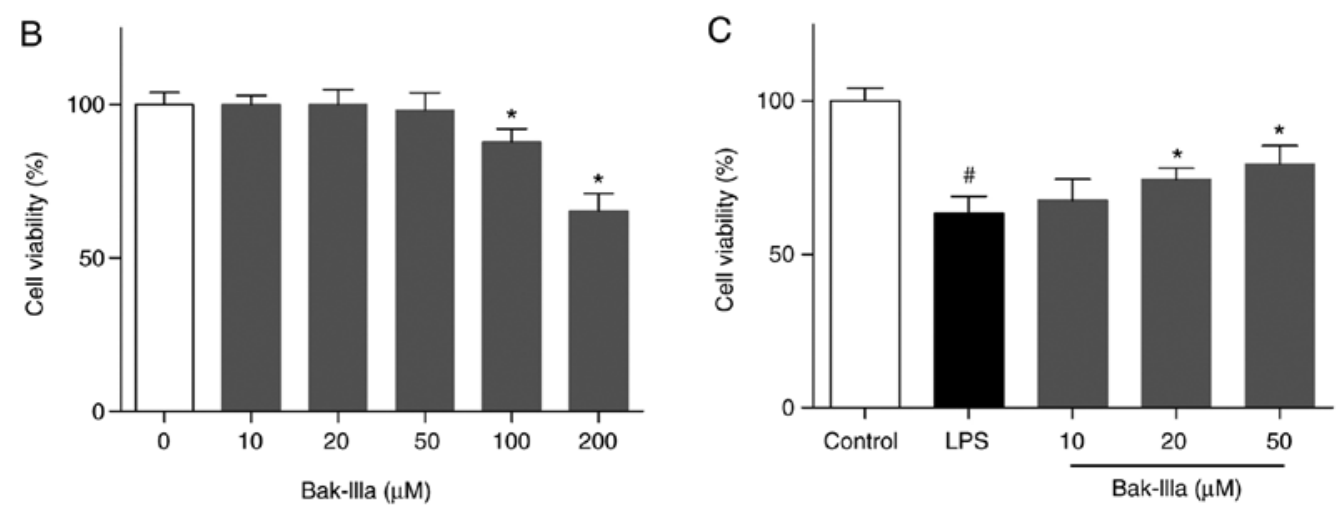

Figure 1. Bak-IIIa alleviates LPS-induced survival inhibition in HUVECs. (A) Molecular structure of Bak-IIIa. (B) Effect of 0, 10, 20, 50, 100 and $200 \mu \mathrm{M}$ Bak-IIIa on the viability of HUVECs. ${ }^{*} \mathrm{P}<0.05$ vs. 0 group. (C) Effect of $0,10,20$ and $50 \mu \mathrm{M}$ Bak-IIIa on the viability of LPS-injured HUVECs. ${ }^{\prime \prime} \mathrm{P}<0.05$ vs. control group; "P<0.05 vs. LPS group. Bak-IIIa, bakkenolide-IIIa; HUVECs, human umbilical vein endothelial cells; LPS, lipopolysaccharide.

between two groups were determined using Student's t-test, whereas one-way analysis of variance and Tukey's post hoc test were used to compare three or more groups. $\mathrm{P}<0.05$ was considered to indicate a statistically significant difference.

\section{Results}

Bak-IIIa ameliorates the LPS-induced inflammatory damage in HUVECs. Initially, the cytotoxic effects exerted by Bak-IIIa on HUVECs were assessed (Fig. 1A). MTT assays indicated that the viability of HUVECs was not significantly changed by treatment with lower concentrations $(10-50 \mu \mathrm{M})$ of Bak-IIIa, whereas viability was reduced by $12-35 \%$ by high doses (100 and $200 \mu \mathrm{M})$ (Fig. 1B). Hence, low doses of Bak-IIIa (10-50 $\mu \mathrm{M})$ were selected for use in subsequent studies. HUVECs were then exposed to LPS, and the results of the MTT assay indicated that the survival of HUVECs was decreased to $\sim 63 \%$ compared with the control group (Fig. 1C). Following further treatment with 20 and $50 \mu \mathrm{M}$ Bak-IIIa, the viability of damaged HUVECs was increased by $\sim 11$ and $\sim 16 \%$, respectively (Fig. $1 \mathrm{C}$ ).

ELISA revealed that the levels of TNF- $\alpha$, IL- $1 \beta$, IL- 8 and IL-6 were significantly increased following LPS-induced stimulation compared with in the control group, indicating that inflammatory damage was inflicted on the HUVECs (Fig. 2A-D). The LPS-injured HUVECs were then treated with 10-50 $\mu \mathrm{M}$ Bak-IIIa, and the results indicated that the levels of the four proinflammatory factors were gradually decreased in response to increasing Bak-IIIa concentrations (Fig. 2A-D). Of the three dose groups, the 20 and $50 \mu \mathrm{M}$ groups induced 

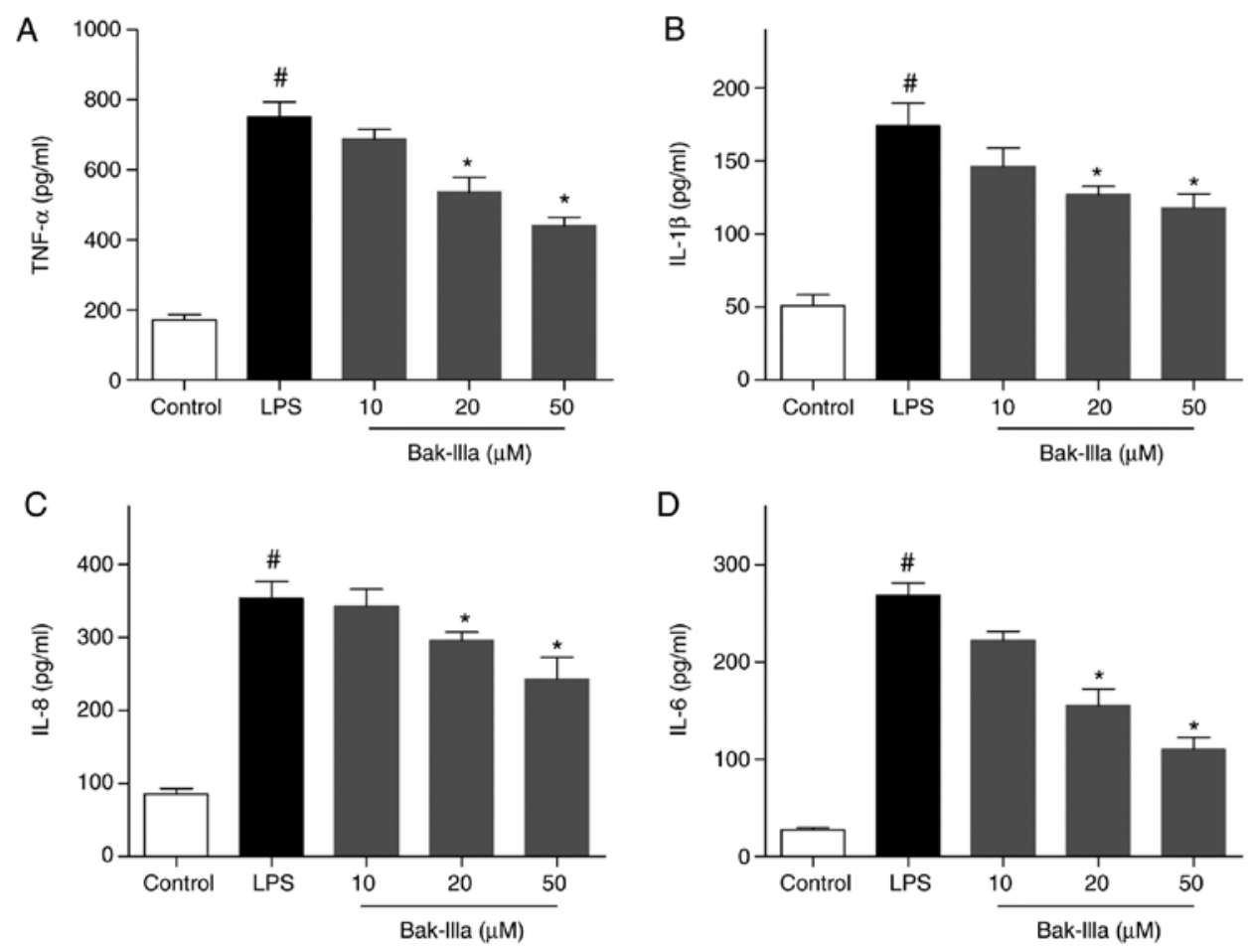

Figure 2. Bak-IIIa ameliorates LPS-induced inflammatory damage in HUVECs. Effect of 0, 10, 20 and $50 \mu \mathrm{M}$ Bak-IIIa on the levels of (A) TNF- $\alpha$, (B) IL-1 $\beta$, (C) IL-8 and (D) IL-6 in LPS-injured HUVECs. " $\mathrm{P}<0.05$ vs. control group; ${ }^{\mathrm{P}}<0.05$ vs. LPS group. Bak-IIIa, bakkenolide-IIIa; HUVECs, human umbilical vein endothelial cells; IL, interleukin; LPS, lipopolysaccharide.

a significant reduction in TNF- $\alpha$, IL-1 $\beta$, IL-8 and IL-6 levels compared with in the LPS group (Fig. 2A-D), thus suggesting that Bak-IIIa treatment was effective in ameliorating LPS-induced inflammatory damage in HUVECs.

Microarray analysis. In order to investigate the mechanisms underlying the effect of Bak-IIIa on HUVECs damaged by inflammation, cells from the LPS and Bak-IIIa groups were subjected to IncRNA microarray analyses, which identified 70 DELs in these two groups. A volcano plot showed the presence of 29 significantly upregulated (red dots) and 41 significantly downregulated (blue dots) DELs in HUVECs that had undergone LPS-induced inflammatory damage and subsequent Bak-IIIa treatment (Fig. 3). These 70 DELs were then ranked according to FC abs values in descending order; the top 10 are listed in Table II. The FC abs of most DELs was $~ 4$, whereas the lncRNA ENST00000441971 had the highest FC abs of $\sim 7$.

IncRNA target prediction and bioinformatics analyses. To obtain a better understanding of the DELs, the cis- and trans-targets were predicted. As a result, 52 cis-regulated targets were obtained from 44 DELs (Table SI), whereas 386 trans-regulated targets (111 overlaps among all 497 rows) were predicted from only 12 DELs (Table SII); nine of the 12 DELs, listed in Table III, covered a total of 382 targets (some overlapped). In addition, the trans-target number for NONHSAT089168, NR_015451 and lnc-MUC20-9:1 reached 100 (Table III).

Among the top 30 targets found to be enriched according to GO analysis, some cis-targets were involved in ion homeostasis and 'autophagy' (Fig. S1), whereas trans-targets were enriched in 'cis-Golgi network' and 'IL-12 production' (Fig. S2). KEGG pathway analysis showed that cis-targets were mainly enriched

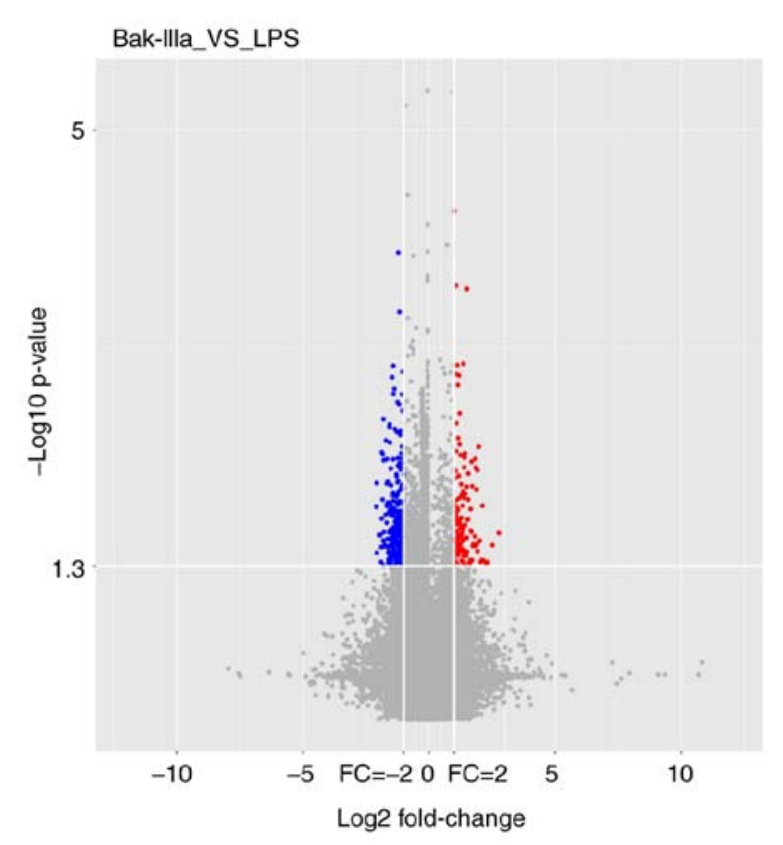

Figure 3. Volcano plot of differentially expressed long non-coding RNAs in LPS-damaged human umbilical vein endothelial cells treated with Bak-IIIa. Bak-IIIa, bakkenolide-IIIa; FC, fold change; LPS, lipopolysaccharide.

in the 'AMPK signaling pathway', whereas several targets were involved in the 'p53 signaling pathway' (Fig. 4). trans-targets were enriched in 'purine metabolism' and 'glycosylphosphatidylinositol-anchor biosynthesis’ (Fig. S3).

RT-qPCR validation of the DELs corresponding to inflammation-related trans-targets. The key words 'inflammation' 
Table II. Top 10 differentially expressed lncRNAs after lipopolysaccharide-damaged human umbilical vein endothelial cells were treated with bakkenolide-IIIa.

\begin{tabular}{lllllll}
\hline Number & \multicolumn{1}{c}{ IncRNA } & P-value & FC & FC (abs) & Regulation & Source \\
\hline 1 & ENST00000441971 & 0.026 & 6.807 & 6.807 & Up & ENSEMBL_GENCODE \\
2 & lnc-NR2F1-8:1 & 0.048 & 4.810 & 4.810 & Up & lncipedia \\
3 & ENST00000609511 & 0.015 & 4.343 & 4.343 & Up & ENSEMBL_GENCODE \\
4 & NONHSAT089168 & 0.045 & 4.250 & 4.250 & Up & NONCODE \\
5 & ENST00000451766 & 0.039 & 0.239 & 4.190 & Down & ENSEMBL_GENCODE \\
6 & NONHSAT113888 & 0.005 & 3.920 & 3.920 & Up & NONCODE \\
7 & ENST00000618397 & 0.037 & 3.788 & 3.788 & Up & ENSEMBL_GENCODE \\
8 & lnc-COMTD1-1:1 & 0.006 & 0.281 & 3.560 & Down & lncipedia \\
9 & lnc-MOK-1:2 & 0.030 & 0.284 & 3.523 & Down & lncipedia \\
10 & NR_015451 & 0.034 & 3.320 & 3.320 & Up & RefSeq
\end{tabular}

abs, absolute; FC, fold change; lncRNA, long non-coding RNA.

Table III. Nine differentially expressed lncRNAs covering 382 trans-targets.

\begin{tabular}{llcllll}
\hline Number & \multicolumn{1}{c}{ IncRNA } & Trans-target number & FC & FC (abs) & Regulation & Sources \\
\hline 1 & NONHSAT089168 & 113 & 4.250 & 4.250 & Up & NONCODE \\
2 & NR_015451 & 154 & 3.320 & 3.320 & Up & RefSeq \\
3 & NR_027411 & 10 & 2.650 & 2.650 & Up & RefSeq \\
4 & NR_033350 & 10 & 2.578 & 2.578 & Up & RefSeq \\
5 & NR_033353 & 10 & 2.435 & 2.435 & Up & RefSeq \\
6 & NR_038275 & 13 & 2.395 & 2.395 & Up & RefSeq \\
7 & lnc-MUC20-9:1 & 100 & 2.366 & 2.366 & Up & lncipedia \\
8 & NR_034072 & 29 & 2.188 & 2.188 & Up & RefSeq \\
9 & lnc-HSP90B1-3:1 & 54 & 2.037 & 2.037 & Up & lncipedia \\
\hline
\end{tabular}

abs, absolute; FC, fold change; lncRNA, long non-coding RNA.

Table IV. Inflammation-related GO terms and trans-targets.

\begin{tabular}{llcl}
\hline GO ID & \multicolumn{1}{c}{ Description } & Count & \multicolumn{1}{c}{ Trans-targets } \\
\hline GO:0006954 & Inflammatory response & 17 & $\begin{array}{l}\text { XIAP, CCR6, CCL5, MEFV, ACER3, OPRM1, IDO1, AXL, } \\
\text { TLR10, REL, LYZ, CCL22, NLRP12, HMGB1, LDLR, } \\
\text { CAMK1D, F2R }\end{array}$ \\
GO:0050729 & $\begin{array}{l}\text { Positive regulation of inflammatory } \\
\text { response } \\
\text { Regulation of inflammatory response }\end{array}$ & 4 & IDO1, NLRP12, TLR10, LDLR \\
GO:0050727 & 7 & & \\
\hline GO, Gene Ontology. & & \\
\hline
\end{tabular}

and 'inflammatory' were searched during GO and KEGG analyses of trans-targets (Tables SIII and SIV), and three inflammation-related GO terms, 'inflammatory response', 'positive regulation of inflammatory response', and 'regulation of inflammatory response', involving a total of 17 targets (some overlapped) were screened (Table IV). The corresponding DELs are listed in Table V, and nine (XIAP, CCL5,
ACER3, OPRM1, IDO1, LYZ, HMGB1, LDLR and F2R) of the 17 targets were those of LINC00294. RT-qPCR indicated that the expression levels of most of the six candidate DELs were consistent with those revealed by the results of microarray analyses. Subsequently, NR_015451 (also termed LINC00294), which displayed the highest verified expression level, was selected for further evaluation (Fig. 5). 


\section{Top 30 of Pathway Enrichment}

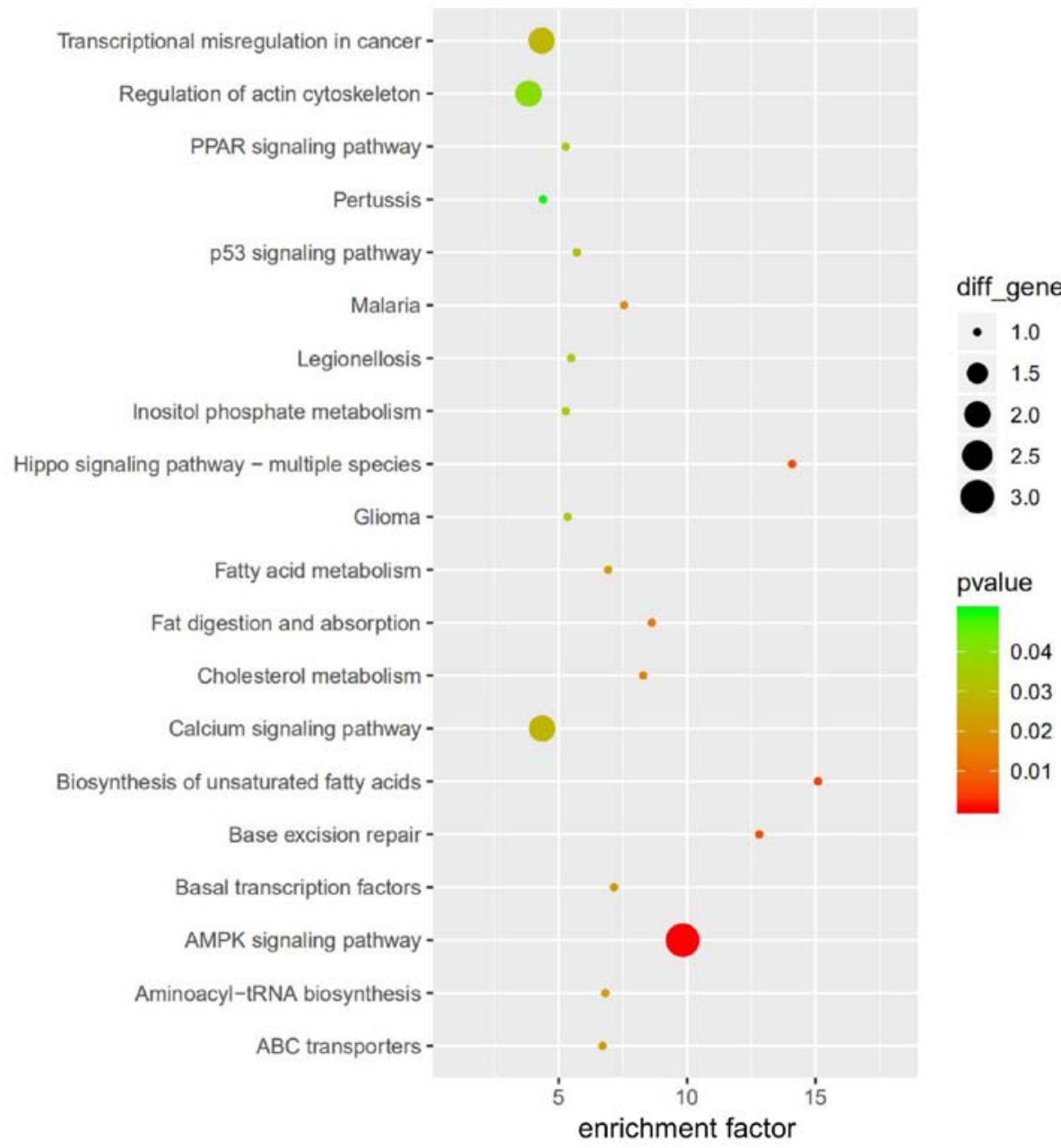

Figure 4. Kyoto Encyclopedia of Genes and Genomes pathway enrichment analysis of the cis-targets of differentially expressed long non-coding RNAs.

Table V. The 17 trans-targets and corresponding lncRNAs.

\begin{tabular}{ll}
\hline Trans-targets & \multicolumn{1}{c}{ lncRNAs } \\
\hline XIAP & NONHSAT089168, NR_015451 \\
CCR6 & Inc-MUC20-9:1 \\
CCL5 & lnc-MUC20-9:1, NR_015451,NR_034072 \\
MEFV & Inc-HSP90B1-3:1 \\
ACER3 & lnc-MUC20-9:1, NR_015451 \\
OPRM1 & NR_015451 \\
IDO1 & NONHSAT089168, NR_015451 \\
AXL & NR_034072 \\
TLR10 & NONHSAT089168 \\
REL & NR_038275 \\
LYZ & NR_015451 \\
CCL22 & lnc-HSP90B1-3:1 \\
NLRP12 & Inc-MUC20-9:1, NONHSAT089168 \\
HMGB1 & NONHSAT089168, NR_015451 \\
LDLR & NR_015451 \\
CAMK1D & lnc-MUC20-9:1 \\
F2R & NR_015451 \\
\hline
\end{tabular}

lncRNA, long non-coding RNA.

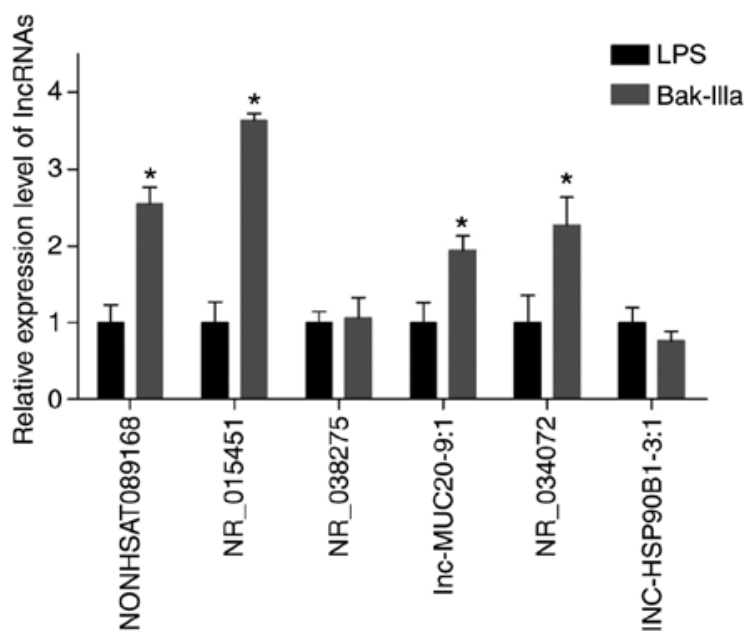

Figure 5. Reverse transcription-quantitative PCR validation of six candidate differentially expressed lncRNAs. NR_015451 is also known as LINC00294. "P<0.05 vs. LPS group. Bak-IIIa, bakkenolide-IIIa; lncRNA, long non-coding RNA; LPS, lipopolysaccharide.

Overexpression of LINC00294 alleviates the LPS-induced inflammatory damage in HUVECs. The RT-qPCR results indicated that LINC00294 was successfully overexpressed 


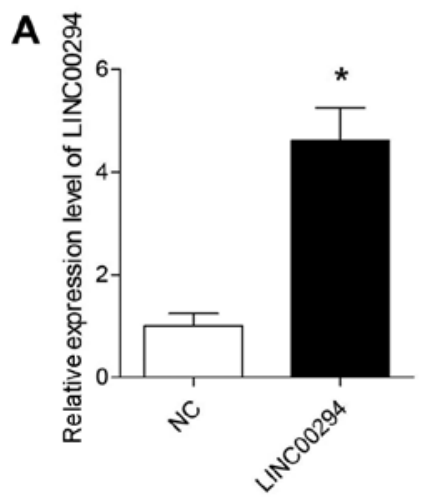

B

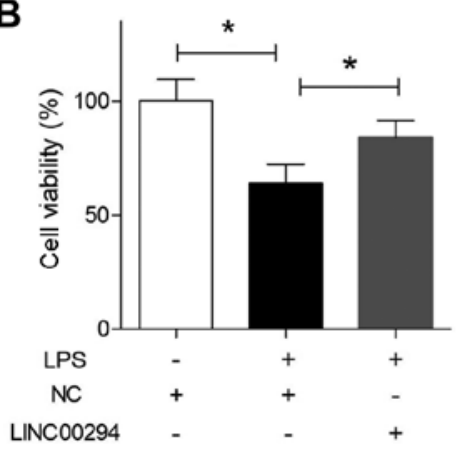

C
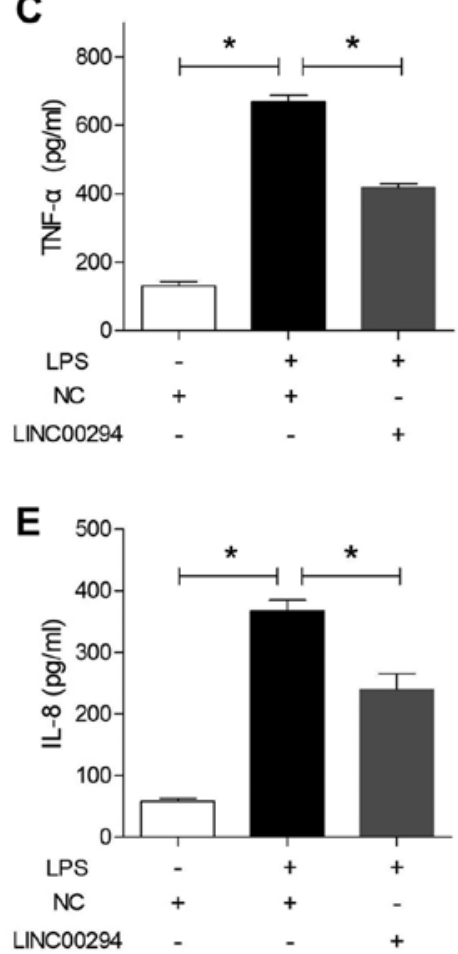
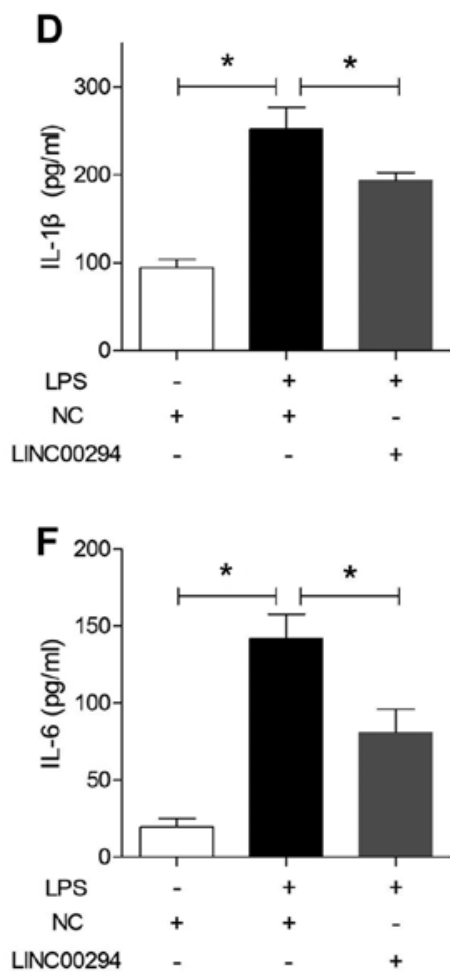

Figure 6. Overexpression of LINC00294 alleviates LPS-induced inflammatory damage in HUVECs. (A) Reverse transcription-quantitative PCR analysis of LINC00294 expression levels in HUVECs overexpressing LINC00294. "P<0.05 vs. NC group. (B) Effect of overexpressed LINC00294 on the viability of LPS-injured HUVECs. *P<0.05. Effect of overexpressed LINC00294 on the levels of (C) TNF- $\alpha$, (D) IL-1 $\beta$, (E) IL-8 and (F) IL-6 in LPS-injured HUVECs. ${ }^{*} \mathrm{P}<0.05$. Bak-IIIa, bakkenolide-IIIa; HUVECs, human umbilical vein endothelial cells; IL, interleukin; LPS, lipopolysaccharide; NC, negative control.

in HUVECs post-transfection with the LINC00294 plasmid (Fig. 6A). Subsequently, cells were treated with LPS and subjected to an MTT assay, which indicated that overexpression of LINC00294 significantly reversed the survival inhibition induced by LPS, compared with cells in the NC group (Fig. 6B). Furthermore, ELISA indicated that the levels of the four proinflammatory factors TNF- $\alpha$, IL-1 $\beta$, IL- 8 and IL- 6 were all significantly decreased in HUVECs overexpressing LINC00294 (Fig. 6C-F), suggesting that upregulation of LINC00294 significantly alleviated the inflammatory damage in HUVECs induced by LPS.

\section{Discussion}

To the best of our knowledge, the present study was the first to assess the effects of Bak-IIIa treatment on LPS-induced inflammatory damage in HUVECs and the lncRNAs involved. The present results indicated that Bak-IIIa ameliorated inflammatory damage in HUVECs.

Vascular inflammation is usually caused by endothelial injury, and thus, prevention of endothelial injury may be considered a key step toward limiting the development of vascular inflammatory processes. LPS acts as a common exogenous factor that stimulates endothelial injury (21). Firstly, LPS treatment generally increases cell permeability and impairs the endothelial barrier, which constitutes an essential step toward the initiation of inflammation $(22,23)$. Secondly, exposure of endothelial cells to LPS leads to inflammation via the production of proinflammatory cytokines/chemokines, cell adhesion molecules and nitric oxide (NO) (24). Hence, LPS-induced inflammatory damage in HUVECs has been widely used as a model for developing potentially effective compounds, as well as for exploring the underlying mechanisms (25). In the present study, HUVECs were exposed to LPS, following which the levels of TNF- $\alpha$, IL- $1 \beta$, IL- 8 and IL- 6 were all significantly enhanced, thereby substantiating the results of previous studies $(26,27)$. TNF- $\alpha$ and IL-1 $\beta$ are two predominant proinflammatory factors involved in the pathogenesis of vascular inflammation. Firstly, increased levels of TNF- $\alpha$ may promote the expression of the inducible form of $\mathrm{NO}$ synthase, which in turn can lead to excessive synthesis of NO, resulting in endothelial dysfunction, apoptosis and vascular damage (28). Secondly, IL-1 $\beta$ can stimulate the synthesis of monocyte chemoattractant protein 1 , resulting in the adhesion of monocytes and the subsequent induction of proliferative, proinflammatory vascular smooth muscle cell phenotypes, thereby initiating the pathological progression of vascular inflammation (29). In the present study, the levels of TNF- $\alpha$ and IL-1 $\beta$, as well as two other proinflammatory factors, IL-8 and IL-6, were all significantly decreased following the treatment of HUVECs with Bak-IIIa, which effectively inhibited vascular inflammation.

In order to clarify the potential mechanism underlying the effect of Bak-IIIa on damaged HUVECs, the DELs present in the two groups were analyzed and 70 were identified. lncRNAs may regulate gene expression in a cis-or trans-acting manner (30). The difference between these two mechanisms is that cis-acting lncRNAs generally influence the expression of nearby genes, whereas trans-acting lncRNAs usually leave the transcription site to execute an array of cellular functions by 
interacting with promoters, enhancers or other proteins (30). Therefore, as trans-targets of lncRNAs are always abundant, potential ways of interacting with these are diverse. The IncRNA target predictions in the present study indicated that 12 DELs covered a total of 386 trans-targets, suggesting a predominant role for these 12 DELs in the amelioration of inflammatory damage in HUVECs by Bak-IIIa. Furthermore, a bioinformatics-based analysis of trans-targets was performed to determine the DELs that may be potentially involved. However, the GO and KEGG pathway enrichment analysis results only indicated those that were significant $(\mathrm{P}<0.5)$; thus, it was difficult to establish certain associations between these and inflammation. Therefore, 'inflammation' and 'inflammatory' were searched in the bioinformatics analyses of trans-targets; this resulted in the screening out of 17 involved targets and six candidate DELs.

Further validation via RT-qPCR and ELISA indicated that upregulation of LINC00294 in the Bak-IIIa group was an important mechanism underlying the ameliorative effect of Bak-IIIa on LPS-induced survival inhibition and inflammatory damage in HUVECs. Among the 17 inflammation-related targets, nine were targets of LINC00294. C-C motif chemokine ligand 5 (CCL5) is a key proinflammatory chemokine expressed in endothelial cells, smooth muscle cells and macrophages, which has been reported to activate various inflammation-related pathological processes, including those of vascular inflammation $(31,32)$. CCL5 may be involved in emodin-alleviated cytotoxicity and high glucose-induced inflammatory events in HUVECs (33), indicating that CCL5 may potentially regulate viability and inflammatory damage in HUVECs. Furthermore, high mobility group box 1 (HMGB1), a ubiquitously expressed nuclear protein, has been reported to serve a predominantly proinflammatory role in the progression of inflammation (34). Several studies confirmed that HMGB1 may have an important regulatory role in vascular endothelial inflammation $(35,36)$. Therefore, it may reasonably be surmised that CCL5 and HMGB1 serve a role in Bak-IIIa-alleviated survival inhibition and inflammatory damage in HUVECs; however, this requires further study.

Although this study focused on the trans-targets of DELs, KEGG pathway-based enrichment analyses of cis-targets provided further leads regarding potential mechanisms that may be involved. AMPK acts as a pivotal cellular energy sensor in the regulation of bioenergy metabolism (37), and exerts anti-inflammatory and antioxidative effects (38). AMPK is widely expressed in the endothelium, and modulates vascular functions via both endothelium-dependent and -independent mechanisms (39). Previous reports have indicated that activation of the AMPK signaling pathway could serve an important role in the amelioration of endothelial inflammation and barrier dysfunction $(40,41)$. Hence, the AMPK signaling pathway and the cis-targets adrenoceptor alpha 1A, tuberous sclerosis 2 and stearoyl-CoA desaturase (SCD) that are enriched in it, as well as the corresponding DELs, lnc-PNMA2-5:1, ENST00000570072 and lnc-SCD-1:8, may serve a role in the alleviation of inflammatory damage in HUVECs via Bak-IIIa.

To date, studies focusing on Bak-IIIa are very limited, and one previous study revealed that the protective effect of Bak-IIIa against cerebral damage were induced through the inactivation of $\mathrm{NF}-\kappa \mathrm{B}$ signaling (10), which is not only involved in the pathogenesis of several diseases but also has an important role in transcriptional regulation. Increasing evidence has uncovered that $\mathrm{NF}-\kappa \mathrm{B}$ could be the downstream effector of lncRNAs, but in some instances, NF- $\kappa \mathrm{B}$ may also be able to regulate lncRNA expression (42). For example, cardiac and apoptosis-related lncRNA was revealed to be translocated to the cytoplasm after $\mathrm{NF}-\kappa \mathrm{B}$ activation in macrophages, and its expression levels were increased in an $\mathrm{NF}-\kappa \mathrm{B}$ dependent manner (43). NF- $\kappa \mathrm{B}$ has also been shown to bind to the HOX antisense intergenic RNA promoter and induce its expression by 16-fold in ovarian cancer cells leading to the maintenance of DNA damage response (44). Moreover, other transcription factors, including p53 and FOXC1, have been reported to regulate $\operatorname{lncRNA}$ expression in a direct or indirect manner $(45,46)$. Hence, the increased level of LINC00294 in injured-HUVECs treated with Bak-IIIa may be attributed to the potential regulatory role of $\mathrm{NF}-\kappa \mathrm{B}, \mathrm{p} 53$ or other transcription factors.

There are some limitations in the present study. First, apoptosis often occurs alongside inflammation in HUVECs stimulated by LPS (47); however, the role of Bak-IIIa in apoptosis of injured HUVECs was not investigated. Secondly, the underlying mechanisms by which Bak-IIIa induced the expression of LINC00294 and how LINC00294 protected HUVECs remain unknown; therefore, further studies are required.

In conclusion, the present findings indicated that Bak-IIIa ameliorated LPS-induced inflammatory damage in HUVECs by upregulating LINC00294, and that Bak-IIIa may have potential as a therapeutic agent against vascular inflammation.

\section{Acknowledgements}

Not applicable.

\section{Funding}

This work was supported by the Project from Shanghai Natural Science Fund Project (grant no. 19ZR1449000), the Training Program of Shanghai Tongji Hospital (grant no. GJPY1812), the National Natural Science Foundation of China (grant no. 81702872) and the Program of Outstanding Young Scientists of Tongji Hospital of Tongji University (grant no. HBRC1808).

\section{Availability of data and materials}

The datasets used and/or analyzed during the current study are available from the corresponding author on reasonable request.

\section{Authors' contributions}

CF and SY worked on the conception and design of the study. JX and HF performed the experiments. JX, LM and HT collected and analyzed data. CF, SY and JX confirmed the authenticity of all the raw data. All authors contributed to the preparation of the manuscript, and read and approved the final manuscript. 


\section{Ethics approval and consent to participate}

Not applicable.

\section{Patient consent for publication}

Not applicable.

\section{Competing interests}

All authors declare that they have no competing interests.

\section{References}

1. Roth GA, Johnson C, Abajobir A, Abd-Allah F, Abera SF, Abyu G, Ahmed M, Aksut B, Alam T, Alam K, et al: Global, regional, and national burden of cardiovascular diseases for 10 causes, 1990 to 2015. J Am Coll Cardiol 70: 1-25, 2017.

2. Wolf MP and Hunziker P: Atherosclerosis: Insights into vascular pathobiology and outlook to novel treatments. J Cardiovasc Transl Res 13: 744-757, 2020.

3. Tabas I, Garcia-Cardeña G and Owens GK: Recent insights into the cellular biology of atherosclerosis. J Cell Biol 209: 13-22, 2015.

4. Libby P, Buring JE, Badimon L, Hansson GK, Deanfield J, Bittencourt MS, Tokgözoğlu L and Lewis EF: Atherosclerosis Nat Rev Dis Primers 5: 56, 2019.

5. Meng G, Liu Y, Lou C and Yang H: Emodin suppresses lipopolysaccharide-induced pro-inflammatory responses and NF- $\kappa \mathrm{B}$ activation by disrupting lipid rafts in CD14-negative endothelial cells. Br J Pharmacol 161: 1628-1644, 2010.

6. Zeng XK, Guan YF, Remick DG and Wang X: Signal pathways underlying homocysteine-induced production of MCP-1 and IL-8 in cultured human whole blood. Acta Pharmacol Sin 26: 85-91, 2005.

7. Moroni F, Ammirati E, Norata GD, Magnoni M and Camici PG: The role of monocytes and macrophages in human atherosclerosis, plaque neoangiogenesis, and atherothrombosis. Mediators Inflamm 2019: 7434376, 2019.

8. Pei L, Dai D, Bao Y, Chen F, Zheng J, Li J, Liu S and Chen X: Determination of bakkenolide $\mathrm{A}$ in rat plasma using liquid chromatography/tandem mass spectrometry and its application to a pharmacokinetic study. J Mass Spectrom 47: 962-968, 2012.

9. Wang YL, Li RP, Guo ML, Zhang G, Zhang N and Ma YL: Bakkenolides from Petasites tricholobus and their neuroprotective effects related to antioxidant activities. Planta Med 75 230-235, 2009

10. Jiang Q, Li RP, Tang Y, Wang YQ, Liu C and Guo ML: Bakkenolide-IIIa protects against cerebral damage via inhibiting NF- $\kappa$ B activation. CNS Neurosci Ther 21: 943-952, 2015

11. Skaper SD, Facci L, Zusso M and Giusti P: An inflammation-centric view of neurological disease: Beyond the neuron. Front Cell Neurosci 12: 72, 2018.

12. Aryal B and Suárez Y: Non-coding RNA regulation of endothelial and macrophage functions during atherosclerosis. Vascul Pharmacol 114: 64-75, 2019.

13. Zhang HN, Xu QQ, Thakur A, Alfred MO, Chakraborty M, Ghosh A and Yu XB: Endothelial dysfunction in diabetes and hypertension: Role of microRNAs and long non-coding RNAs. Life Sci 213: 258-268, 2018.

14. Zhu X, Chen D, Liu Y, Yu J, Qiao L, Lin S, Chen D, Zhong G, Lu X, Wang Y, et al: Long noncoding RNA HOXA-AS3 integrates NF- $\mathrm{KB}$ signaling to regulate endothelium inflammation. Mol Cell Biol 39: e00139-19, 2019.

15. Cao L,Zhang Z,Li Y,Zhao P and Chen Y: LncRNA H19/miR-let-7 axis participates in the regulation of ox-LDL-induced endothelial cell injury via targeting periostin. Int Immunopharmacol 72 496-503, 2019

16. Zhu X, Liu Y, Yu J, Du J, Guo R, Feng Y, Zhong G, Jiang Y and Lin J: LncRNA HOXA-AS2 represses endothelium inflammation by regulating the activity of NF- $\mathrm{BB}$ signaling. Atherosclerosis 281: 38-46, 2019.

17. Li S, Sun Y, Zhong L, Xiao Z, Yang M, Chen M, Wang C, Xie X and Chen X: The suppression of ox-LDL-induced inflammatory cytokine release and apoptosis of HCAECs by long non-coding RNA-MALAT1 via regulating microRNA-155/SOCS1 pathway. Nutr Metab Cardiovasc Dis 28: 1175-1187, 2018.
18. Casper J, Zweig AS, Villarreal C, Tyner C, Speir ML, Rosenbloom KR, Raney BJ, Lee CM, Lee BT, Karolchik D, et al: The UCSC genome browser database: 2018 update. Nucleic Acids Res 46D: D762-D769, 2018

19. Tafer H and Hofacker IL: RNAplex: A fast tool for RNA-RNA interaction search. Bioinformatics 24: 2657-2663, 2008.

20. Livak KJ and Schmittgen TD: Analysis of relative gene expression data using real-time quantitative PCR and the 2(-Delta Delta C(T)) method. Methods 25: 402-408, 2001

21. Song L, Kang C, Sun Y, Huang W, Liu W and Qian Z: Crocetin inhibits lipopolysaccharide-induced inflammatory response in human umbilical vein endothelial cells. Cell Physiol Biochem 40: 443-452, 2016

22. Yin X, Liang Z, Yun Y and Pei L: Intravenous transplantation of BMP2-transduced endothelial progenitor cells attenuates lipopolysaccharide-induced acute lung injury in rats. Cell Physiol Biochem 35: 2149-2158, 2015.

23. Uhlig S, Yang Y, Waade J, Wittenberg C, Babendreyer A and Kuebler WM: Differential regulation of lung endothelial permeability in vitro and in situ. Cell Physiol Biochem 34: 1-19, 2014.

24. Kim JH, Jeong JH, Jeon ST, Kim H, Ock J, Suk K, Kim SI, Song KS and Lee WH: Decursin inhibits induction of inflammatory mediators by blocking nuclear factor-kappaB activation in macrophages. Mol Pharmacol 69: 1783-1790, 2006.

25. Zhang M, Pan H, Xu Y, Wang X, Qiu Z and Jiang L: Allicin decreases lipopolysaccharide-induced oxidative stress and inflammation in human umbilical vein endothelial cells through suppression of mitochondrial dysfunction and activation of $\mathrm{Nrf2}$. Cell Physiol Biochem 41: 2255-2267, 2017.

26. Hou X, Yang S and Yin J: Blocking the REDD1/TXNIP axis ameliorates LPS-induced vascular endothelial cell injury through repressing oxidative stress and apoptosis. Am J Physiol Cell Physiol 316: C104-C110, 2019.

27. Makó V, Czúcz J, Weiszhár Z, Herczenik E, Matkó J, Prohászka Z and Cervenak L: Proinflammatory activation pattern of human umbilical vein endothelial cells induced by IL-1 $\beta$, TNF- $\alpha$, and LPS. Cytometry A 77: 962-970, 2010.

28. Mangoni AA, Zinellu A, Sotgia S, Carru C, Piga M and Erre GL: Protective effects of methotrexate against proatherosclerotic cytokines: A review of the evidence. Mediators Inflamm 2017: 9632846, 2017.

29. Khan R, Rheaume E and Tardif JC: Examining the role of and treatment directed at IL-1 $\beta$ in atherosclerosis. Curr Atheroscler Rep 20: 53, 2018.

30. Kopp F and Mendell JT: Functional classification and experimental dissection of long noncoding RNAs. Cell 172: 393-407, 2018.

31. Marques RE, Guabiraba R, Russo RC and Teixeira MM: Targeting CCL5 in inflammation. Expert Opin Ther Targets 17: 1439-1460, 2013.

32. Nosalski R and Guzik TJ: Perivascular adipose tissue inflammation in vascular disease. Br J Pharmacol 174: 3496-3513, 2017.

33. Gao Y, Zhang J, Li G, Xu H, Yi Y, Wu Q, Song M, Bee YM, Huang L, Tan M, et al: Protection of vascular endothelial cells from high glucose-induced cytotoxicity by emodin. Biochem Pharmacol 94: 39-45, 2015.

34. Sachdev U and Lotze MT: Perpetual change: Autophagy, the endothelium, and response to vascular injury. J Leukoc Biol 102: 221-235, 2017.

35. Cai W, Duan XM, Liu Y, Yu J, Tang YL, Liu ZL, Jiang S, Zhang CP, Liu JY and Xu JX: Uric acid induces endothelial dysfunction by activating the HMGB1/RAGE signaling pathway. Biomed Res Int 2017: 4391920, 2017.

36. Tang ST, Wang F, Shao M, Wang Y and Zhu HQ: MicroRNA-126 suppresses inflammation in endothelial cells under hyperglycemic condition by targeting HMGB1. Vascul Pharmacol 88: 48-55, 2017.

37. Kim WH, Lee JW, Suh YH, Lee HJ, Lee SH, Oh YK, Gao B and Jung MH: AICAR potentiates ROS production induced by chronic high glucose: Roles of AMPK in pancreatic beta-cell apoptosis. Cell Signal 19: 791-805, 2007.

38. Bijland S, Mancini SJ and Salt IP: Role of AMP-activated protein kinase in adipose tissue metabolism and inflammation. Clin Sci (Lond) 124: 491-507, 2013.

39. Ewart MA and Kennedy S: AMPK and vasculoprotection. Pharmacol Ther 131: 242-253, 2011.

40. Hu R, Wang MQ, Ni SH, Wang M, Liu LY, You HY, Wu XH, Wang YJ, Lu L and Wei LB: Salidroside ameliorates endothelial inflammation and oxidative stress by regulating the AMPK/NF- $\kappa \mathrm{B} / \mathrm{NLRP} 3$ signaling pathway in AGEs-induced HUVECs. Eur J Pharmacol 867: 172797, 2020. 
41. Dennhardt S, Finke KR, Huwiler A and Coldewey SM: Sphingosine-1-phosphate promotes barrier-stabilizing effects in human microvascular endothelial cells via AMPK-dependent mechanisms. Biochim Biophys Acta Mol Basis Dis 1865: 774-781, 2019.

42. Gupta SC, Awasthee N, Rai V, Chava S, Gunda V and Challagundla KB: Long non-coding RNAs and nuclear factor- $\kappa \mathrm{B}$ crosstalk in cancer and other human diseases. Biochim Biophys Acta Rev Cancer 1873: 188316, 2020.

43. Castellanos-Rubio A, Kratchmarov R, Sebastian M, Garcia-Etxebarria K, Garcia L, Irastorza I and Ghosh S: Cytoplasmic form of carlr lncRNA facilitates inflammatory gene expression upon NF- $\kappa \mathrm{B}$ activation. J Immunol 199: 581-588, 2017.

44. Özeş AR, Miller DF, Özeş ON, Fang F, Liu Y, Matei D, Huang T and Nephew KP: NF- $\kappa \mathrm{B}$-HOTAIR axis links DNA damage response, chemoresistance and cellular senescence in ovarian cancer. Oncogene 35: 5350-5361, 2016.
45. Chen S, Thorne RF,Zhang XD,WuMand LiuL:Non-codingRNAs, guardians of the p53 galaxy. Semin Cancer Biol: Sep 11, 2020 (Epub ahead of print). doi: 10.1016/j.semcancer.2020.09.002

46. Sun CC, Zhu W, Li SJ, Hu W, Zhang J, Zhuo Y, Zhang H, Wang J, Zhang Y, Huang SX, et al: FOXC1-mediated LINC00301 facilitates tumor progression and triggers an immune-suppressing microenvironment in non-small cell lung cancer by regulating the HIF1 $\alpha$ pathway. Genome Med 12: 77, 2020

47. Jung TW, Park HS, Choi GH, Kim D, Ahn SH, Kim DS, Lee T and Jeong JH: Maresin 1 attenuates pro-inflammatory reactions and ER stress in HUVECs via PPAR $\alpha$-mediated pathway. Mol Cell Biochem 448: 335-347, 2018.

This work is licensed under a Creative Commons Attribution-NonCommercial-NoDerivatives 4.0 International (CC BY-NC-ND 4.0) License. 